unpleasant symptoms of hypothyroidism. Differentiated thyroid tumours may continue to secrete $\mathrm{Tg}$ despite continued suppressive treatment with thyroxine; the finding of high serum $\mathrm{Tg}$ values in such patients may obviate the need to stop thyroxine before carrying out the investigation. This aspect is being studied further.

Dr Serge C Ng Tang Fui is supported by a research grant from the Guy's Hospital Special Trustees.

Requests for reprints should be addressed to: Dr M N Maisey, Department of Nuclear Medicine and Endocrinology, Guy's Hospital, London SE1 9RT.

\section{References}

1 Van Herle, A J, et al, fournal of Clinical Investigation, 1973, 52, 1320.

2 Van Herle, A J, and Uller, R P, fournal of Clinical Investigation, 1975, $56,272$.

${ }^{3}$ Shlossberg, A H, Jacobson, J C, and Ibbertson, H K, Clinical Endocrinology, 1979, 10, 17.

4 Schneider, A B, et al, Annals of Internal Medicine, 1977, 86, 29.

${ }^{5}$ Lo Gerfo, P, et al, Lancet, 1977, 1, 881.

${ }^{6}$ Maisey, M N, and Ng Tang Fui, S C, in Thyroid Cancer, ed W Duncan. Berlin, Springer-Verlag. In press.

7 Pochin, E E, Clinical Radiology, 1967, 18, 113.

${ }^{8}$ Edmonds, C J, et al, British Fournal of Radiology, 1977, 50, 799.

(Accepted 28 fune 1979)

\title{
Randomised trial comparing buprenorphine and diamorphine for chest pain in suspected myocardial infarction
}

\author{
M J HAYES, A R FRASER, J R HAMPTON
}

British Medical fournal, 1979, 2, 300-302

\section{Summary and conclusions}

Buprenorphine, a new powerful analgesic agent, was used to treat chest pain in patients with suspected myocardial infarction. Initial studies showed no significant changes in systemic or pulmonary artery blood pressure or in heart rate after intravenous buprenorphine. Sublingual buprenorphine also appeared effective in relieving pain, but its onset of action was considerably delayed compared with the intravenous route. A randomised double-blind controlled trial of equivalent doses of buprenorphine and diamorphine showed no significant difference between the drugs in terms of pain relief and duration of action. The occurrence of nausea, vomiting, and other side effects was similar in the two groups. The onset of action of buprenorphine was slightly but significantly slower than that of diamorphine.

Since buprenorphine seems to be comparable with diamorphine in action and is not a controlled drug, it may prove useful in both general and hospital practice.

\section{Introduction}

In many respects diamorphine is an ideal drug for relieving the pain of myocardial infarction. It is a powerful analgesic, its effect is rapid, and it is free of adverse haemodynamic side effects. Unfortunately it is addictive and therefore unpopular, particularly with general practitioners. Attempts to find equally powerful but non-addictive analgesic drugs have been unsuccessful; in particular, pentazocine was found to cause pulmonary and systemic vasoconstriction and was therefore unsuitable for patients with heart disease. ${ }^{1}$

Department of Medicine, University Hospital, Nottingham

M J HAYES, DM, MRCP, senior medical registrar

A R FRASER, MB, MRCP, senior house officer

J R HAMPTON, DM, FRCP, consultant physician and reader in medicine
Buprenorphine (Temgesic, Reckitt \& Colman) is a synthetic compound derived from thebaine, which has been found effective in relieving postoperative pain. ${ }^{2}$ It has recently been released as a non-controlled drug. We describe here a study on the haemodynamic effects of buprenorphine in patients with myocardial infarction, a comparison of the action of intravenous and sublingual routes of administration, and also a double-blind controlled study comparing intravenous buprenorphine and diamorphine in the relief of chest pain due to suspected myocardial infarction.

\section{Patients and methods}

Three studies were performed.

Study 1-Haemodynamic studies were performed on an initial 10 patients with myocardial infarction proved on electrocardiography (ECG). All had received diamorphine previously but then required further analgesia for recurrent pain. The pulmonary artery pressure was recorded continuously before and after an intravenous injection of $0.3 \mathrm{mg}$ buprenorphine, by means of a $3 \mathrm{~F}$ gauge polyethylene catheter inserted percutaneously via an antecubital vein. Cuff measurements of the systemic blood pressure were made at defined intervals. The ECG was monitored continuously and measurements of heart rate obtained from the ECG.

Study 2-Forty-three patients who required analgesia in the coronary care unit (CCU) were given either injections of intravenous buprenorphine or sublingual tablets. Eighteen received a total of 20 tablets of sublingual buprenorphine $0.4 \mathrm{mg}$, and 25 received a total of 40 injections of intravenous buprenorphine $0.3 \mathrm{mg}$ as and when they needed analgesia for chest pain. In this group only systemic blood pressure and heart rate were measured and the ECGs were continuously monitored. The degree of pain relief and more particularly the time of onset of pain relief was assessed subjectively by the medical and nursing staff.

Study 3-One hundred and twenty patients who were admitted to the CCU with chest pain due to suspected myocardial infarction and who required analgesia were randomly allocated in a double-blind fashion to receive either buprenorphine $0.3 \mathrm{mg}$ intravenously or diamorphine $5 \mathrm{mg}$ intravenously. There were no medical contraindications for inclusion in this trial. Patients were randomised in blocks of six, the trial ampoules being prepared and issued by the General Hospital pharmacy daily because of the instability of diamorphine when in solution. After entry into the trial records were kept of the time, dose, and frequency of subsequent analgesic adminis- 
tration. The time, degree, and duration of pain relief were monitored using an unmarked visual analogue scale, ${ }^{3}$ which was scored by the patient. The scale was subsequently measured and pain relief expressed as a percentage of the original score. If the patients were asleep they were left undisturbed and considered to have complete pain relief. The incidence of nausea, vomiting, and other adverse reactions was also recorded.

\section{Results}

Intravenous buprenorphine did not produce any significant change in heart rate or systemic diastolic blood pressure (studies 1 and 2) or pulmonary artery pressure; fig 1 shows the mean results from the patients in study 1 . There was a sustained fall in systemic arterial systolic pressure of about $10 \mathrm{~mm} \mathrm{Hg}$, but at no time did this reach a statistically significant level-for example, at one hour $t=1.4191$; $\mathbf{P}<0 \cdot 1$.

Sublingual buprenorphine had no significant effect on heart rate and systemic blood pressure and in most patients provided good pain relief (study 2), but intravenous buprenorphine produced considerably faster pain relief (table I).

Table II compares the patients in study 3 who were randomly allocated to receive either intravenous buprenorphine $0.3 \mathrm{mg}$ or intravenous diamorphine $5 \mathrm{mg}$. There were no significant differences between the groups in terms of age, sex ratio, previous angina or infarction, the duration of chest pain, mean serum aspartate aminotransferase (AST) and $\alpha$-hydroxybutyrate dehydrogenase (SHBD) concentrations, or in the number with different sites of myocardial infarction as shown by ECG. The heart rate and systemic systolic and diastolic blood pressures on admission to the CCU were likewise similar in the two groups. Twelve patients in the buprenorphine group and 15 patients in the diamorphine group were eventually found to have inconclusive evidence of myocardial infarction. One patient in each group had inadequate records and both were excluded from the study.

Table II also shows that in each group 32 patients had received

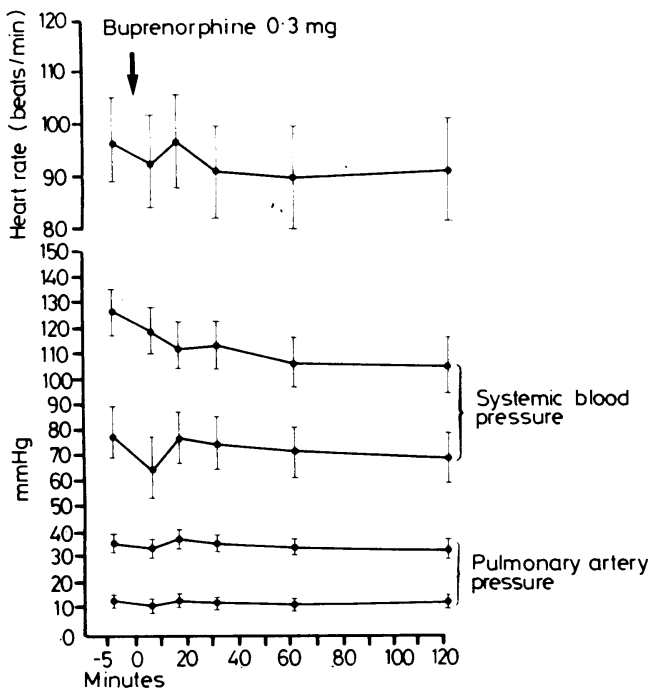

FIG 1-Haemodynamic changes after intravenous buprenorphine in 10 patients. Valves are means $\pm S E$ of means.

TABLE I-Time of complete pain relief after buprenorphine (cumulative total)

\begin{tabular}{lcc}
\hline & \multicolumn{2}{c}{ No of administrations of: } \\
\cline { 2 - 3 } $\begin{array}{c}\text { Complete pain relief } \\
\text { obtained after: }\end{array}$ & $\begin{array}{c}\text { Intravenous } \\
\text { buprenorphine } \\
0 \cdot 3 \mathrm{mg}\end{array}$ & $\begin{array}{c}\text { Sublingual } \\
\text { buprenorphine } \\
0 \cdot 4 \mathrm{mg}\end{array}$ \\
\hline 5 Minutes & 9 & 2 \\
15 Minutes & 30 & 4 \\
30 Minutes & 33 & 16 \\
45 Minutes & 1 & 19 \\
\hline Inadequate pain relief & 19 & 1 \\
\hline
\end{tabular}

TABLE II-Comparison of groups of patients randomly allocated to receive either intravenous buprenorphine or diamorphine. Values are means $\pm S D$

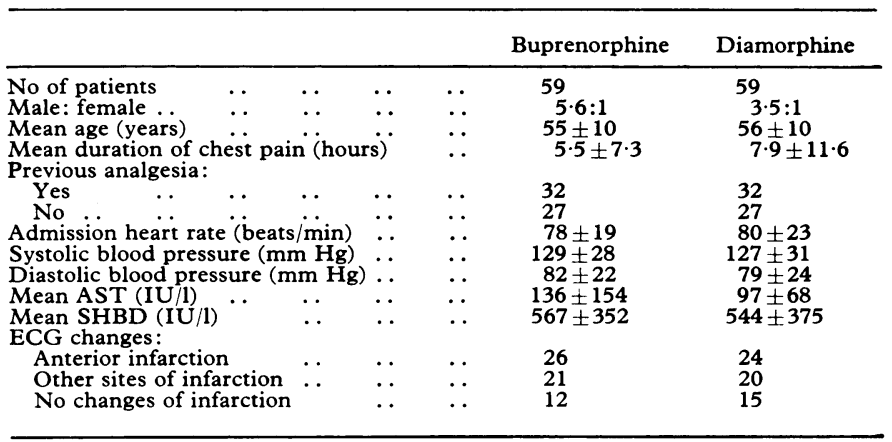

TABLE III-Need for further analgesia after administration of buprenorphine or diamorphine

\begin{tabular}{lccc}
\hline & & Buprenorphine & Diamorphine \\
\hline No further analgesia required $\ldots$ & $\ldots$ & 27 & 23 \\
Further analgesia within 6 hours & $\ldots$ & 12 & 16 \\
Further analgesia at 6-48 hours & $\ldots$ & 16 & 16 \\
\hline Total* & & 55 & 55 \\
\hline
\end{tabular}

*Excluding patients who died.

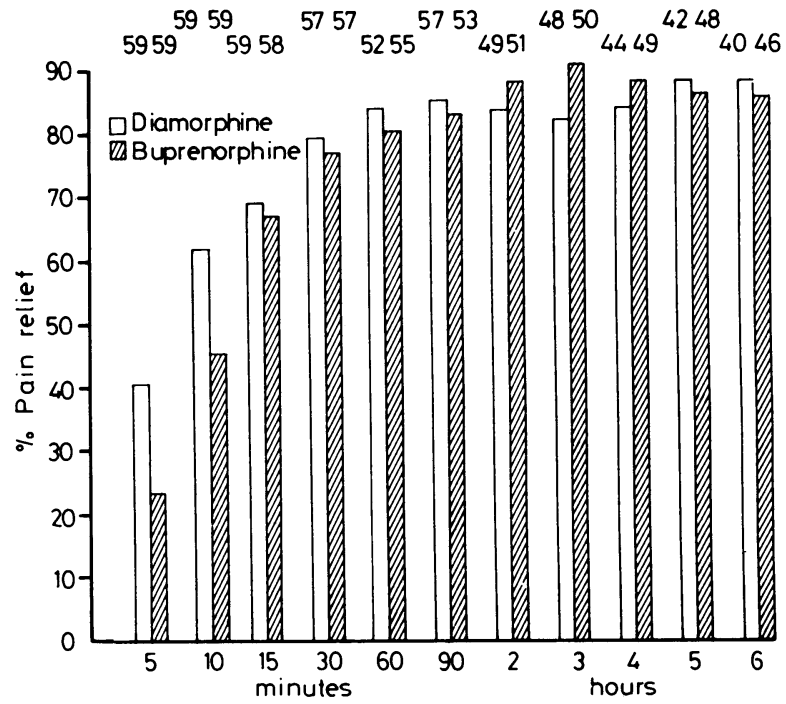

FIG 2-Percentage pain relief after intravenous buprenorphine and diamorphine. Numbers above columns indicate number of patients remaining in the study at each time, the remainder having died or having been withdrawn because of the need for further analgesia.

analgesia before admission to the CCU, either from their general practitioner or in the casualty department of the General Hospital; the drugs given were morphine, diamorphine, or pethidine. The mean time of entry into the trial from previous analgesic administration was similar in the two groups.

Table III shows the subsequent requirement for analgesia in the groups of patients initially treated with buprenorphine or diamorphine. During the six-hour period of recording pain relief using the visual analogue scale 12 patients in the buprenorphine group and 16 patients in the diamorphine group had incomplete pain relief and needed additional diamorphine. This difference was not statistically significant, nor was there any significant difference between the groups in terms of total requirement for analgesia during the whole 48 hours during which the patients were in the CCU. Four patients in each group died within 48 hours of admission to the CCU, one patient in the buprenorphine group and three in the diamorphine group dying within the initial six-hour assessment period.

Measurements of the visual analogue scale showed that the mean starting pain score was similar in the two groups. Fig 2 compares the 
effects of buprenorphine and diamorphine on the degree of pain relief expressed as a percentage of the start score. At five minutes the percentage pain relief in the buprenorphine group was significantly less than in the diamorphine group $(P<0.01)$, but the difference progressively diminished so that both groups were similar at 15 minutes. No difference was then apparent between the two groups to the completion of the six-hour period.

Among the patients who survived and who needed no further analgesia more who received buprenorphine had long periods of sleep than those given diamorphine; 25 out of 50 patients and 15 out of 48 respectively were asleep at three hours, but otherwise there were no other apparent differences in the two groups. In particular, no major side effects were experienced with either drug. Twelve patients who received buprenorphine and seven patients who received diamorphine vomited during the six-hour study period, but this difference was not statistically significant.

In a comparison between buprenorphine and diamorphine given to those patients who had received no prior analgesic there was again no difference between the two drugs in terms of subsequent analgesic requirement or percentage pain relief.

\section{Discussion}

In healthy individuals buprenorphine causes a fall in both systolic and diastolic arterial pressure but does not affect the heart rate. ${ }^{+}$We also found a fall in systemic arterial pressure, particularly systolic pressure, but the change was not great and was of a similar magnitude to that often seen after diamorphine. No other adverse haemodynamic effects were noted, and, in particular, buprenorphine did not appear to induce the peripheral and pulmonary effects of pentazocine. ${ }^{1}$ Haemodynamically, therefore, buprenorphine appears ideal for patients with myocardial infarction and does not necessitate particular monitoring.

Our initial subjective assessment of the route of administration showed that buprenorphine was effective as an analgesic when given either intravenously or sublingually, but the latter route considerably delayed the onset of pain relief. Accurate and objective assessment of pain relief is difficult, particularly in a busy CCU. We therefore based our assessment on the patient's subsequent analgesic requirement and on his own assessment of his pain relief using a visual analogue scale. The unmarked visual analogue scale seems to be the most accurate simple approach to a subject's assessment of his degree of pain relief and is probably better than a graded scale. ${ }^{3}$ Using these scales we could detect a slight but definite delay in the onset of action of buprenorphine compared with diamorphine. Though not of major clinical importance, this does suggest that the visual analogue scale can discriminate differences in pain relief caused by different drugs.

Apart from the slight delay in the onset of action, buprenorphine provided the same high degree of pain relief as diamorphine and this effect was sustained for at least six hours in most patients. There was no difference between the two drugs in the incidence of nausea and vomiting and no major side effects were observed.

We conclude that buprenorphine is a powerful analgesic, comparable with diamorphine, that can be safely administered to patients with suspected myocardial infarction. Although buprenorphine is more expensive than diamorphine, the fact that it is not a controlled drug makes it attractive for use in both hospital and general practice.

We thank Mrs J Walls and Mr J Wilson of the General Hospital pharmacy, the nurses on the CCU, Dr A Ward of Reckitt and Colman Limited; and the consultant medical staff of the General Hospital for allowing us to treat patients in their care.

\section{References}

1 Jewitt, D E, Maurer, B J, and Hubner, P J B, British Medical fournal, 1970, 1, 795 .

2 Howell, B D, British fournal of Anaesthesia, 1977, 49, 913.

${ }^{3}$ Huskinsson, E C, Lancet, 1974, 2, 1127.

${ }^{4}$ Orwin, J M, Pain: New Perspectives in Management and Measurement, p 141. Edinburgh, Churchill Livingstone, 1977.

(Accepted 25 fune 1979)
ONE HUNDRED YEARS AGO Deputy Surgeon-General Logie, late of the Royal Horse Guards, has recently called attention to the necessity of suitable means for dressing a wound being placed securely in the tunic of every officer and soldier proceeding on service against an enemy. He suggests that every tunic should have a long narrow pocket inside the lining along the spine, or rather made of the lining itself, about two-and-a-half inches wide, extending from the lower edge of the collar to the buttons at the waist; and that every officer and man should have in his possession a made-up packet containing about two-and-a-half yards of carbolised bandage, some carbolised gauze, and some carbolised ligature-thread, encased in a piece of thin waterproof sheeting or oiled silk, folded so as to fit the long narrow pocket neatly. This is to be slipped into the pocket in the tunic, and stitched firmly to the edge of the collar before the man goes into action. The surgeon would then find all that is requisite on his patient, or on a dead man near him, by ripping open a few threads of the collar of the tunic. Dr Logie objects to the official "soldiers' first field-dressing," as it is called, because it is placed in the knapsack, and knapsacks are often thrown aside, and so the dressing is apt to be lost. On the other hand, if placed in the tunic this cannot happen, and by having the pocket along the spine, the dressing is not likely to be even carried into a wound; while, if neatly fitted, it cannot be discovered by even a martinet on parade. It certainly does seem better that the dressing should be carried in the soldier's tunic than in his valise or knapsack. It was carried in a breast-pocket on the left side of the tunic during the Ashantee war of 1873-74, and was then reported to answer its purpose very satisfactorily. It is also carried on the soldiers' person in many Continental armies. Another suggestion which has been made by $\mathrm{Dr}$ Ward Cousins of Southsea in connection with "immediate surgery on the battle-field," is that every soldier should be instructed in some simple method of dressing a wound and in the use of a tourniquet.
In addition to the supply of materials for a first field-dressing, he recommends that a small elastic belt or leathern strap and pad should be fixed round the soldier's helmet or his arm, in such a way that it could be readily undone and easily applied with one hand to a wounded part for the purpose of arresting haemorrhage. Soldiers, perhaps, are not so apt as sailors for dealing with sudden emergencies; but with the simple means required for a primary dressing always at hand, we imagine there is not likely to be any difficulty in having them applied, if not by the wounded man himself, by a comrade, in case of the absence of a surgeon or trained orderly. Still, if some plain instructions were given systematically on the subject, as suggested by Dr Cousins, and these were kept within the bounds of the private soldier's capacity, the plan would probably be attended with good results. We have heard of army surgeons giving lessons on such subjects to both officers and men while on board-ship on the way to active service in the field, and that this teaching has been greatly appreciated at the time, and has proved of much utility afterwards. There seems to be no valid reason why what has proved advantageous in particular instances should not be adopted as a general rule in the military service. The other suggestions, however, that every soldier should be furnished with an elastic tourniquet, is one which will not be acceptable to many surgeons. The subject of a general distribution of tourniquets among soldiers in armies, which has been not unfrequently advocated, is very fully discussed in Professor Longmore's work on Gunshot Injuries in the section on their general treatment in field practice; and the conclusion come to is that any such universal issue of these instruments would be likely to be followed by a vast amount of positive mischief. We would recommend, therefore, that the objections which have been urged in the work above mentioned should be carefully considered before a general issue of tourniquets among officers and soldiers in the ranks is advocated by their professional advisers. (British Medical fournal, 1879.) 